\title{
Boron nanotubes and their properties: semiempirical investigation
}

\author{
I.V. Zaporotskova ${ }^{a}$, E.V. Perevalova and N.P. Zaporotskova
}

Volgograd State University (VolSU), Volgograd, Russia

\begin{abstract}
The problem of formation possibility nanotubular structures is actively discussed now. We considered the fragments of single-wall boron nanotubes $(n, n)(n=4,5,6,9,11,12)$. Calculations were carried out by IB-CCC method [1]. The analysis of band-gap showed that all of them are semiconductors. Energy of deformation decreases with increase of the diameter of B-tubes $(n, n)$. We considered the B-nanotubes $(n, 0)(n=4,5,6,8,12)$. In this case deformation energy is increases with increase of the diameter of tubes $(n, 0)$. Calculations of boron tube $(6,6)$ which contained various defects of structure were obtained by the semiempirical MNDO scheme. We research substitution imperfection of $\mathrm{B}$ atom by atom $\mathrm{C}$, ions $\mathrm{C}^{+}, \mathrm{C}^{-}$. We found out substitution energy of defects and its energy level. We studied the B-tube with atomic vacancy and determined the energy of defect activation and the relative portion of vacancies.At the moment active search of new surface structures capable of effective adsorption of different gases is being carried out. We have investigated an binding opportunity between the $\mathrm{H}, \mathrm{F}, \mathrm{O}, \mathrm{Cl}$ atoms and the outer surface of B-nanotube $(6,6)$ and have studied the mechanism of this process. The calculations are carried out with the use of quantum chemical MNDO scheme. Regular hydrogenation of boron nanotubes was investigated. We can confirm that generation of gas-phase hydrogen composite materials based on boron nanotube is possible.
\end{abstract}

\section{Semiempirical investigation of boron nanotubes and some structure- modification composites on its base}

Last decades the boron became a very important element. Boron and its chemical compounds started to be used in nuclear energy and rocket technology, metallurgy, chemical industry and in the other fields. Now many scientists and tens scientific laboratories study boron and its chemical compounds. It was found out that boric atoms are able to form not only ionic bonds. Boron atoms can bind chains, frame, net and ets [2-4]. However, it is not known much about a boron till now. Nevertheless, boron is the most important element in current technology. The quantity of modification of an elementary boron is not known yet. Therefore, investigation of boron possible configuration is very actual at the present time.

\subsection{Possibility of boron nanotubes creation}

We assumed that boron is the nearest neighbour of carbon and it can make quasiplanar hexogonal form similar to structure of graphite. That's why it is possible to assume the possibility of boron nanotubes formation. We have considered the fragments of single-wall boron nanotubes $(n, n)$ type $(n=4,5,6,9,11,12)$. Calculations were carried out by the Ionic-Building Covalent Cyclic Cluster model (IB-CCC) and semiempirical quantum-chemical MNDO scheme [1]. The analysis of band-gap $\Delta \mathrm{E}_{\mathrm{g}}$ showed that all of them are semiconductors, irrespective of diameter. Energy of deformation was calculated as a difference of quasiplanar extended unit cell (EUC) energy and energy of boron nanotube which made of corresponding EUC. We defined that energy of deformation decreases with increase of the diameter of boron tubes $(n, n)$ type (see fig. 1a). The charge analysis of boron tubes has established that their symmetry is stable (charges on boron atoms are almost equal to zero).

aemail: irinaz@rbcmail.ru

This is an Open Access article distributed under the terms of the Creative Commons Attribution-Noncommercial License (http://creativecommons.org/licenses/by-nc/3.0/), which permits unrestricted use, distribution, and reproduction in any noncommercial medium, provided the original work is properly cited. 
Also we have considered the fragments of single-wall boron nanotubes $(\mathrm{n}, 0)$ type $(\mathrm{n}=4,5,6,8,12)$. In this case energy of deformation is increased with increase of the diameter of boron tubes $(\mathrm{n}, 0)$ type (see fig. $1 \mathrm{~b})$. So, we can conclude that formation processes of «zig-zag» boron nanotube from flat hexogonal boron structure energetically is not useful and possible. The basic electron-energy characteristics of boron nanotubes (n, n) and $(\mathrm{n}, 0)$ type are shown in the table 1.

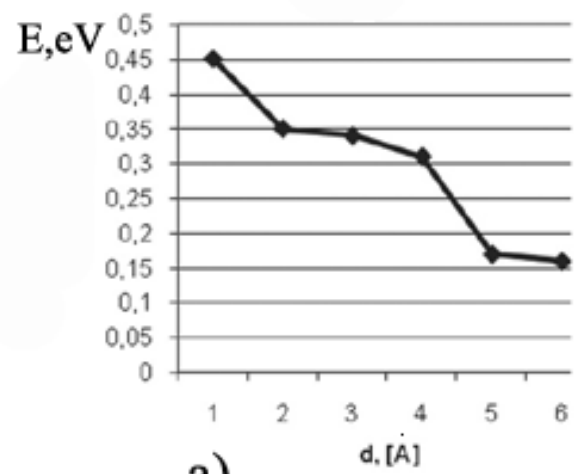

a)

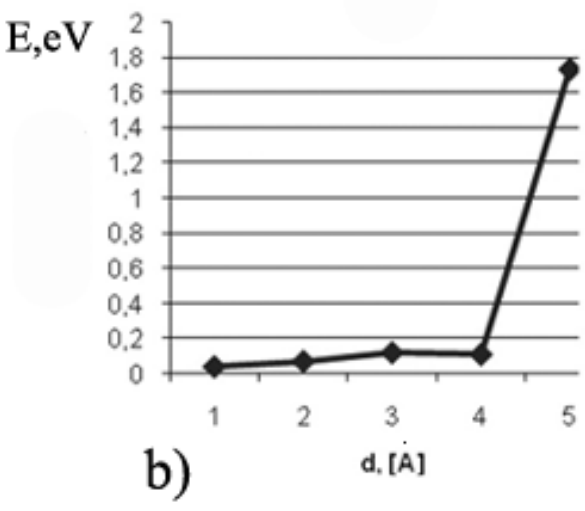

b)

Fig.1. Dependence energy of deformation from diameter a) (n, n)-type; (n, 0)-type of tube

Table 1. The basic electron-energy characteristics of boron nanotubes $(n, n)$ and $(n, 0)$ type: $n$ - number hexagons on perimeter of tube, $\mathrm{d}$ - diameter of tube, $\mathrm{E}_{\mathrm{un}}$ - unit energy density, $\Delta \mathrm{E}_{\mathrm{g}}$ - band-gap energy, $\mathrm{E}_{\mathrm{def}}$ - energy of deformation.

\begin{tabular}{|c|c|c|c|c|c|}
\hline Type of tube & $\mathrm{n}$ & $\mathrm{d}, \AA$ & $\mathrm{E}_{\mathrm{un}}, \mathrm{eV}$ & $\Delta \mathrm{E}_{\mathrm{g},}, \mathrm{eV}$ & $\mathrm{E}_{\text {def }}, \mathrm{eV}$ \\
\hline \multirow{5}{*}{$(\mathrm{n}, \mathrm{n})$} & & & & & \\
\cline { 2 - 6 } & 4 & 5.25 & 68.01 & 0.07 & 0.45 \\
\cline { 2 - 6 } & 5 & 6.90 & 67.91 & 0.04 & 0.35 \\
\cline { 2 - 6 } & 6 & 8.25 & 68.14 & 0.90 & 0.34 \\
\cline { 2 - 6 } & 11 & 12.42 & 68.97 & 0.02 & 0.31 \\
\hline \multirow{5}{*}{$(\mathrm{n}, 0)$} & 12 & 15.18 & 69.34 & 0.35 & 0.17 \\
\cline { 2 - 6 } & 4 & 3.03 & 69.33 & 0.22 & 0.16 \\
\cline { 2 - 6 } & 5 & 3.99 & 67.85 & 0.27 & 0.04 \\
\cline { 2 - 6 } & 6 & 4.77 & 67.88 & 0.00 & 0.07 \\
\cline { 2 - 6 } & 12 & 9.35 & 67.92 & 0.01 & 0.12 \\
\hline
\end{tabular}

\subsection{Boron nanotubes with various defects}

It is known that defects change conduct properties of carbon nanotubes [5, 6]. So, we have decided to study the change of boron tube which contained difference defects. Calculations of boron nanotubes $(6,6)$ which contained various defects of structure (structure-modification B-tubulenes) were obtained by the quantumchemical semiempirical computational MNDO scheme with molecular cluster model use. The geometric structure of $(6,6)$ tubes has been simulated by fragment containing fore boron hexagons along the tube axis and six hexagons along the perimeter. B-B bond length is $1.44 \mathrm{~A}$. The unsaturated cluster boundary chemical bonds have been completed by hydrogen atoms. We research substitution defect of boron atom by carbon atom (C), positive carbon ion $\left(\mathrm{C}^{+}\right)$and negative carbon ion $\left(\mathrm{C}^{-}\right)$. Defects have been situated in the middle of the cluster in order to exclude the influence of boundary effects (see fig.2). 


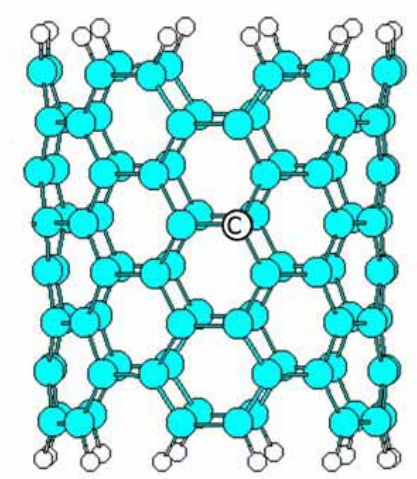

Fig. 2. The cluster of boron nanotubes $(6,6)$ which contained substitution defect

We found out substitution energy of defects and their atomic energy level. And we have seen that the width of forbidden energy band is not change (see fig.3)

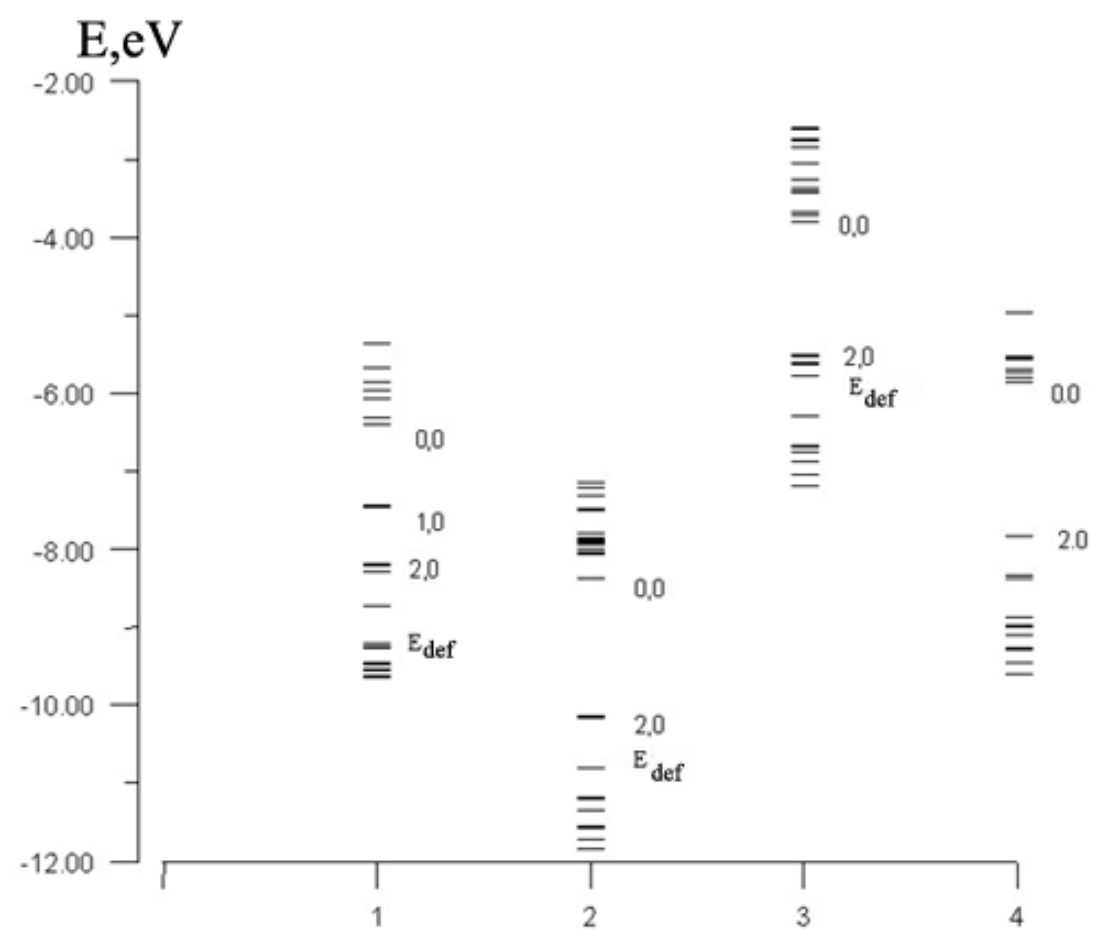

Fig.3. Single-electron energy spectrum of boron nanotubes $(6,6)$ type:

1) substitution imperfection of boron atom by carbon atom;2) substitution imperfection of boron atom by positive carbon ion; 3) substitution imperfection of boron atom by negative carbon ion; 4) flawless boron nanotubes

Then we have studied the boron nanotube with atomic vacancy and determined the energy of defect activation and the relative portion of vacancies. The process of atomic vacancy creation of boron tube has been simulation by step-by-step abstraction of one central boron atom. The result of this process is shown at the graph (see fig. 4). We have seen, that the boron atom should overcome potential energy barrier, which identify like energy of defect activation $\quad E_{a c t}=E_{e b}-E_{v}=0.68 \mathrm{eV}$. This value let calculate the relative portion of vacancies for $\mathrm{T}=1000 \mathrm{~K}$ by the well-known formula [8]:

$$
\mathrm{n} / \mathrm{N} \sim \exp \left[-\mathrm{E}_{\mathrm{act}} / \mathrm{kT}\right]
$$

It equal 0.53 .

The minimum energy $E_{v}$ situated apart 0.7 from atom $B$ to cylindrical surface of tubul. In this case length of B-B bond is changed from $1.44 \AA$ to $1.56 \AA$. It is obvious that such position of surface atom B is stable. 


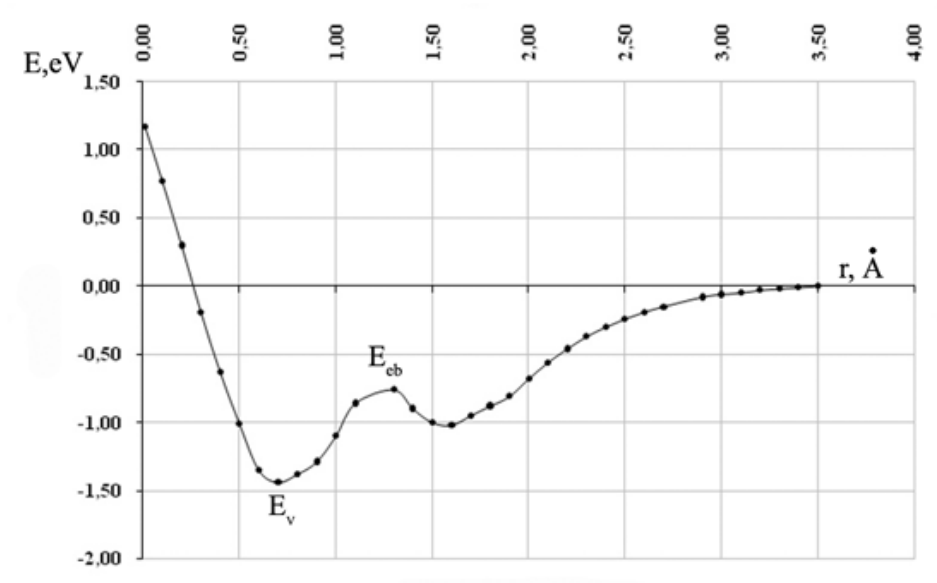

Fig. 4. Potential energy as a function of $B$ atom distance from the $(6,6)$ nanotube surface.

\section{The adsorbtion properties of boron nanotubes}

Sorption properties of tubular structure are object of attention at a wide range of researchers for a long time. Carbon nanotubes possess especially characteristics [5, 6]. Many works are devoted to research adsorption mechanisms of atoms and molecules on tubular surface (internal and external) [7-8]. However, now the great attention is given to research and synthesis of new nanotubular systems which characteristics can give new prospects of their use. Recently research of boron nanotube have begun. Their unique properties can provide their applications in new fields of a science and technology [9].

The problem of superficial structures hydrogenation has great value. These structures can be used as storehouse of molecular hydrogen. Carbon nanotube sated by hydrogen are already experimentally received. However at the moment intensive search of new surface structures capable of effective adsorption of different gases (including hydrogen) is being carried out. Therefore we consider that research sorption properties of Bnanotube is actual.

\subsection{Mechanisms of easy atoms adsorption on a surface boron nanotube $(6,6)$ - type}

We have investigated a binding opportunity between the atoms of hydrogen, fluorine, chlorine, oxygen and the outer surface of single-walled boron nanotube of $(6,6)$ type and have studied the mechanism of this process. The calculations have been carried out within the model of molecular cluster with the use of quantum chemical MNDO scheme [10].

Three variants of adatoms' orientation over the tube surface were considered: I) over boric atom, II) over the centre of B-B bond, III) over hexagon centre (see fig.5).
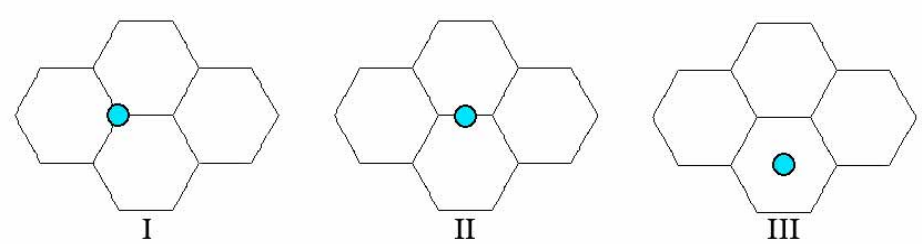

Fig. 5. Three variants of adatoms' orientation over the tube surface: I) over boric atom, II) over the centre of the B-B bond, III) over the hexagon centre.

Surface patterns of potential energy for these processes were built. Atoms attack the boron nanotubes have been simulated by a step-by-step approach for all adatoms orientations. For position (I) the analysis of curves showed that all atoms are adsorbed on the surface of B-tube. Adsorption distances $\left(\mathrm{R}_{\mathrm{ad}}\right)$ and adsorption energy $\left(\mathrm{E}_{\mathrm{ad}}\right)$ were calculated for all selected atoms. Adsorption processes for positions II and III were simulated in a similar manner. It turned out that adsorption of $\mathrm{H}$ and $\mathrm{O}$ atoms is implemented only for position II, and adsorption of $\mathrm{H}$ atom - for position III. Profile of potential energy of adsorption process for atom hydrogen is shown (see fig. 6 - 8). The basic electron-energy characteristics of adsorption process for all atoms $(\mathrm{H}, \mathrm{Cl}, \mathrm{O}, \mathrm{F})$ are presented in the table 2 . 


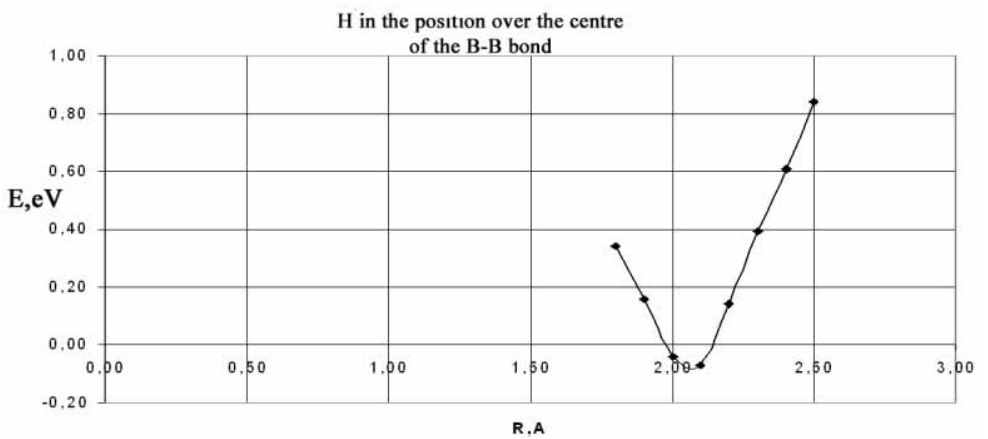

Fig. 6. Profile of potential energy of adsorption process for hydrogen in the position over the centre of the B-B bond $\mathrm{H}$ in the position over the hexagon centre

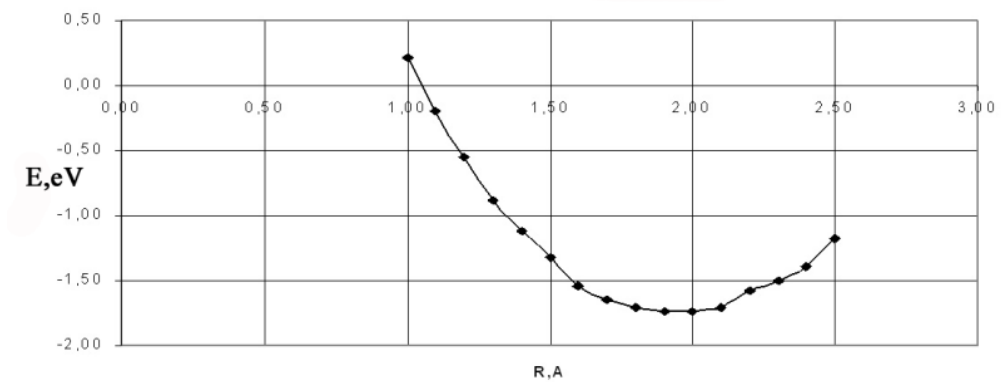

Fig. 7. Profile of potential energy of adsorption process for hydrogen in the position over the hexagon centre $\mathrm{H}$ in the position over boric atom

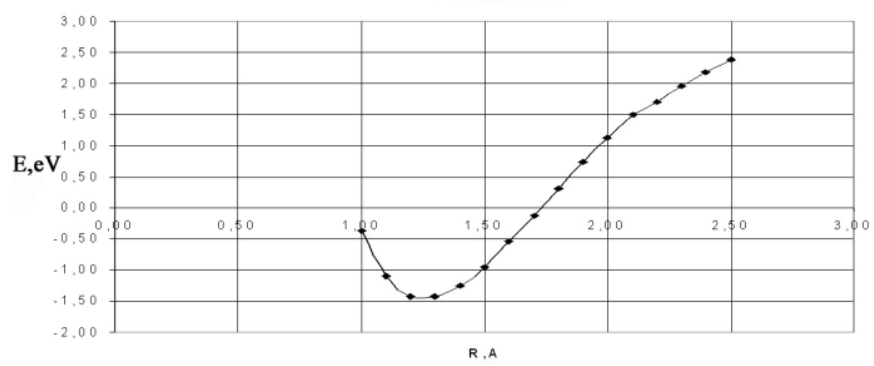

Fig. 8. Profile of potential energy of adsorption process for hydrogen in the position over boric atom

\subsection{Regular hydrogenation of boron nanotubes}

Regular hydrogenation of boron nanotubes have been investigated. Calculations of two variants of hydrogen atoms binding to the outer surface of nanotube were carried out: 1) $\mathrm{H}$ atoms are arranged over B atoms of three adjacent hexagon layers (six $\mathrm{H}$ atoms over each layer) so that rings of superlattice formed by adatoms are not displaced relatively each other; 2) even ring of atatoms are displaced as for uneven at the length of B-B bond (see fig.9). The second position of hydride B-nanotube structure turned out to be energetically more favourable.

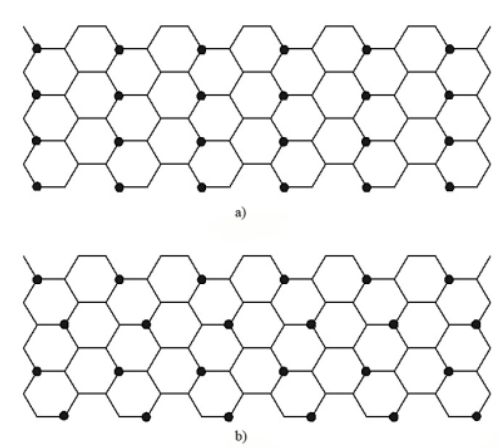

Fig. 9. Regular hydrogenation of boron nanotubes. Unfolded extended unit cell of tubule $(6,6)$-type. a), b) - locations of hydrogen atoms. 
Table 2. The basic electron-energy characteristics of adsorption process for all atoms ( $\mathrm{H}, \mathrm{Cl}, \mathrm{O}, \mathrm{F})$ : I) over boric atom, II) over the centre of the B-B bond, III) over the hexagon centre, $E_{a d}$ - adsorption energy, $R_{a d}$ - adsorption distance, $\Delta E_{g}, e V-$ band-gap energy.

\begin{tabular}{|c|c|c|c|c|}
\hline \multicolumn{2}{|c|}{} & I & II & III \\
\hline \multirow{4}{*}{$\mathrm{Cl}$} & $\mathrm{E}_{\mathrm{a}}, \mathrm{eV}$ & $-0,02$ & - & - \\
\cline { 2 - 5 } & $\mathrm{R}_{\mathrm{a}}, \AA$ & 2 & - & - \\
\cline { 2 - 5 } & $\Delta \mathrm{E}_{\mathrm{g}}, \mathrm{eV}$ & 1,5 & - & - \\
\hline \multirow{4}{*}{$\mathrm{H}$} & $\mathrm{E}_{\mathrm{a}}, \mathrm{eV}$ & $-1,43$ & $-0,07$ & $-1,74$ \\
\cline { 2 - 5 } & $\mathrm{R}_{\mathrm{a}}, \AA$ & 1,2 & 2,1 & 2 \\
\cline { 2 - 5 } & $\Delta \mathrm{E}_{\mathrm{g}}, \mathrm{eV}$ & 1,2 & 1,3 & 1,5 \\
\hline \multirow{4}{*}{$\mathrm{F}$} & $\mathrm{E}_{\mathrm{a}}, \mathrm{eV}$ & $-3,7$ & $-1,64$ & - \\
\cline { 2 - 5 } & $\mathrm{R}_{\mathrm{a}}, \AA$ & 1,4 & 1 & - \\
\cline { 2 - 5 } & $\Delta \mathrm{E}_{\mathrm{g}}, \mathrm{eV}$ & 0,7 & 0,8 & - \\
\cline { 2 - 5 } & $\mathrm{E}_{\mathrm{a}}, \mathrm{eV}$ & 1,86 & - & - \\
\cline { 2 - 5 } & $\mathrm{R}_{\mathrm{a}}, \AA$ & 1,6 & - & - \\
\cline { 2 - 5 } & $\Delta \mathrm{E}_{\mathrm{g}}, \mathrm{eV}$ & 1,3 & - & - \\
\hline
\end{tabular}

\section{Conclusions}

We studied single-wall boron nanotubes $(n, n)$ and $(n, 0)$ types, which have proved to be semiconductors. Also, we found that the process of a «zig-zag» boron nanotube construction from hexagon (quasi) - planar boron structures is energetically unfavourable, and that's why it is very unlikely to occur.

We studied defects of substitution on the surface of a boron nanotube and found that the width of energy gap in a tubule with defects remains stable. We also studied the process of vacancy formation and found the basic characteristics of this process. Boron nanotubes with defects of substitution and vacancies can be considered as structurally-modified composites on the boron nanotube basis.

We have considered a possibility of forming bonds between atoms of hydrogen, fluorine, chlorine, oxygen and the outer surface of single-walled boron nanotube of $(6,6)$ type and have studied the mechanism of this process. We confirmed that the formation of gas-phase composite materials based on the boron nanotube is possible.

Acknowledgements

The authors are grateful to A. Name (ESPCI Paris) and B. Name (E.N.S. Lyon) for useful discussions.

\section{References}

[2] Ivanovskii A.L., Shveikin G.P. Quantum chemistry in the material science. Boron, its alloy and compound. - Ekaterinburg: publishing house "Ekaterinburg", 1997.- 400p. (In Russian)

[4] Xiaobao Yang, Yi Ding, and Jun Ni. Phys. Rev. B 77, 041402(R). - 2008.

[1] Litinskii A.O., Lebedev N.G., Zaporotskova I.V. Journal of physical chemistry - 1995. - V. 69. № 1. P. 189. (In Russian)

[3] H. Tang and S. Ismail-Beigi. Physical Review Letters. - 2007. - T. 99. - C. 115501.

[5] Saito R., Dresselhaus M.S., Dresselhaus G. Physical properties of carbon nanotubes. Imperial College Press. - 1999. - 251 P.

[6] Zaporotskova I.V. Nano- and microsystems technique. - 2005. - № 10. - P. 7 - 18. (In Russian)

[7] Lebedev N.G., Zaporotskova I.V., Chernozatonskii L.A. International Journal of Quantum Chemistry. 2003. - V. 96, № 2. - P. 149 - 154.

[8] Lebedev N.G., Zaporotskova I.V., Chernozatonskii L.A. International Journal of Quantum Chemistry. 2003. - V. 96, № 2. - P. 142 - 148.

[9] Xiaobao Yang, Yi Ding, and Jun Ni. Phys. Rev. B 77, 041402(R). - 2008.

[10] Dewar M.J.S., Thiel W. J. Amer. Chem. Soc. - 1977 - V. 99 - P. 4899 - 4906. 